\title{
Global analysis of histone lysine acetylation and proteomic changes in EC109 cells treated with the histone deacetylase inhibitor FK228
}

\author{
ZHIWEN PAN ${ }^{1}$, MINGLI WANG $^{1}$, ZHEN YE $^{1}$, SHENGJIE ZHANG ${ }^{2}$ and XIAOHONG XU ${ }^{1,2}$ \\ ${ }^{1}$ Clinical Laboratory Department; ${ }^{2}$ Key Laboratory Diagnosis and Treatment Technology on Thoracic Oncology, \\ Zhejiang Cancer Hospital, Hangzhou, Zhejiang 310022, P.R. China
}

Received June 20, 2016; Accepted May 23, 2017

DOI: $10.3892 / \mathrm{ol} .2018 .8312$

\begin{abstract}
FK228 is a selective inhibitor of histone deacetylases that exhibits marked antitumor activity in cancer cells and xenograft models. However, the effect of FK228 on the global profile of histone lysine acetylation and the proteome of EC109 cells remains poorly understood. The present study aimed at analyzing histone lysine acetylation and identifying the proteomic changes in EC109 cells following treatment with FK228, using the stable isotope labelling by amino acids in cell culture technique and a high-sensitivity mass spectrometer. In total, 87 acetylation sites and 3,515 proteins revealed changes in response to FK228 treatment. Of the 87 acetylation sites, 25 were quantifiable and 19 were quantified with ratio of $>1.3$. Notably, no downregulated lysine acetylation (Kac) sites were quantified in the present study and the 62 unquantified Kac sites were only identified in the FK228-treated cells. Bioinformatic analysis revealed that these quantifiable proteins were primarily involved in multiple biological functions and metabolic pathways as well as in protein complexes. The results of the present study revealed the extensive lysine acetylome and proteome in EC109 cells and expanded upon the current understanding of the anticancer mechanism of FK228 in EC109 cells.
\end{abstract}

\section{Introduction}

Esophageal cancer (EC) accounts for between 1 and 3\% of all incidences of cancer occurring in the USA, but exhibits a

Correspondence to: Mrs. Xiaohong Xu, Clinical Laboratory Department, Zhejiang Cancer Hospital, 38 Banshan Road, Hangzhou, Zhejiang 310022, P.R. China

E-mail: zjhzxxh@163.com

Abbreviations: ESCC, esophageal squamous cell carcinoma; HATs, histone acetyltransferases; HDACs, histone deacetylases; FBS, fetal bovine serum; KEGG, Kyoto Encyclopedia of Genes and Genomes; GO, Gene Ontology

Key words: FK228, EC109 cells, histone lysine acetylation, proteome higher incidence in Asia (1). In China, esophageal squamous cell carcinoma (ESCC) is the predominant histological subtype accounting for $\sim 90 \%$ of all EC cases (2). Despite progress in the application of combined chemotherapy and radiotherapy, ESCC remains an intractable cancer. Thus, the development of novel antitumor agents to improve the prognosis of patients with ESCC is urgently required.

Histone acetylation status is controlled by histone acetyltransferases (HATs) and histone deacetylases (HDACs), which are associated with chromatin structure and influence gene transcription (3-5). HATs direct the addition of acetyl groups to lysine residues on histones and regulate gene expression. HDACs remove acetyl groups from histones, silencing the transcription of several oncogenes and tumor suppressor genes. HDAC inhibitors have been revealed to serve a therapeutic role in numerous distinct human cancer cell lines (6). FK228, a novel HDAC inhibitor, has been demonstrated to exhibit antitumor activity in several cancer cells, alone or in combination with other drugs or therapeutic methods $(7,8)$ and was approved by the US Food and Drug Administration in 2009 (9) for use in patients with cutaneous T-cell lymphoma with a Phase II study currently in progress in Italy (10). The molecular mechanisms underlying the antitumor effects of FK228 including apoptosis, growth arrest and angiogenesis inhibition have been reported, and gene expression profiling has been performed to evaluate the effects of FK228 (11-14). However, the effects of FK228 on the global levels of histone acetylation and the proteome of EC109 cells remain poorly understood.

The present study aimed at employing integrated approaches including the stable isotope labeling by amino acids in cell culture (SILAC) technique and mass spectrometry-based quantitative proteomics to quantify the dynamic changes in histone lysine acetylation and in the proteome in one pair of EC109 cells.

\section{Materials and methods}

Cell culture. The ESCC cell line EC109 was acquired from Shanghai Cell Bank (Shanghai, China) and was maintained in Dulbecco's modified Eagle's medium (DMEM; Pierce; Thermo Fisher Scientific, Inc., Waltham, MA, USA) supplemented with $10 \%$ fetal bovine serum (FBS; Gibco; Thermo 
Fisher Scientific, Inc.) at $37^{\circ} \mathrm{C}$ in a humidified atmosphere containing $5 \% \mathrm{CO}_{2}$.

$\left({ }^{13}\right.$ C) Lysine labeling and FK228 treatment of EC109 cells. EC109 cells were grown to $80 \%$ confluence in high glucose (4.5 g/l) DMEM (with glutamine and sodium pyruvate) containing $10 \% \mathrm{FBS}$ and $1 \%$ penicillin-streptomycin at $37^{\circ} \mathrm{C}$ with $95 \%$ air and $5 \% \mathrm{CO}_{2}$. The cells were subsequently labeled with either 'heavy isotopic lysine' $\left[\left({ }^{13} \mathrm{C}\right)\right.$ lysine, $\left({ }^{13} \mathrm{C}_{6},{ }^{15} \mathrm{~N}_{4}\right)$ arginine] or 'light isotopic lysine' $\left[\left({ }^{12} \mathrm{C}\right)\right.$ lysine $]$ for $>6$ generations prior to being harvested to achieve $>97 \%$ labeling efficiency by using a SILAC Protein Quantitation kit (Pierce; Thermo Fisher Scientific, Inc.), according to the manufacturer's protocol. Cells were further cultured in SILAC medium to obtain the desired cell number $\left(\sim 5 \times 10^{8}\right)$ in $15,150-\mathrm{cm}^{2}$ flasks.

The 'light' labeled cells were treated with $43.5 \mathrm{ng} / \mathrm{ml} \mathrm{FK} 228$, and the 'heavy' labeled cells were treated with the equivalent measure of dimethyl sulfoxide. Following treatment, the cells were maintained in SILAC medium for $24 \mathrm{~h}$ before being harvested and washed twice with ice-cold PBS supplemented with $2 \mu \mathrm{M}$ trichostatin A and $30 \mathrm{mM}$ nicotinamide. Following snap-freezing in liquid nitrogen, the cell pellets were obtained and stored in a $-80^{\circ} \mathrm{C}$ freezer for future use.

Histone extraction and trypsin digestion. The harvested 'heavy' and 'light' labeled cells were lysed using 2X NETN buffer (200 mM NaCl, $2 \mathrm{mM}$ EDTA, $100 \mathrm{mM}$ Tris- $\mathrm{HCl}$ and $1.0 \%$ NP-40; pH7.2) supplemented with $0.5 \%$ Triton X-100 on ice for $30 \mathrm{~min}$. The supernatants were retained following centrifugation at $20,000 \mathrm{~g}$ for $10 \mathrm{~min}$ at $4^{\circ} \mathrm{C}$. Next, equal amounts of crude proteins were mixed in the supernatants labeled 'heavy' or 'light', and the crude proteins were then precipitated by adding trifluoroacetic acid (TFA) at a final concentration of $15 \%(\mathrm{v} / \mathrm{v})$ (soluble fraction). The protein pellets were washed twice with $20^{\circ} \mathrm{C}$ acetone prior to being dissolved in $100 \mathrm{mM} \mathrm{NH}_{4} \mathrm{HCO}_{3}(\mathrm{pH}$ 8.0) for trypsin digestion. The remaining cell pellets were dissolved in $8 \mathrm{M}$ urea to extract the chromatin-binding proteins. The protein concentration was measured using a bicinchoninic acid assay kit (Thermo Fisher Scientific, Inc.) and equal amounts of chromatin-binding proteins were added to the urea solution and the proteins were precipitated by adding TFA at a final concentration of $15 \%$ $(\mathrm{v} / \mathrm{v})$ (nuclear pellet fraction). The protein pellets were washed twice with $-20^{\circ} \mathrm{C}$ acetone prior to being dissolved in $100 \mathrm{mM}$ $\mathrm{NH}_{4} \mathrm{HCO}_{3}$ for trypsin digestion.

Trypsin (Promega Corporation, Madison, WI, USA) was added to the protein solution at a trypsin to protein ratio of 1:50 $(\mathrm{w} / \mathrm{w})$ for digestion at $37^{\circ} \mathrm{C}$ for $16 \mathrm{~h}$. DTT was added to obtain a final concentration of $5 \mathrm{mM}$ prior to incubation at $50^{\circ} \mathrm{C}$ for $30 \mathrm{~min}$. Iodoacetic acid was subsequently added to alkylate the proteins and achieve a final concentration of $15 \mathrm{mM}$; this was then followed by incubation at room temperature in the dark for $30 \mathrm{~min}$. The alkylation reaction was quenched by adding $30 \mathrm{mM}$ cysteine (final concentration) at room temperature for another $30 \mathrm{~min}$. Trypsin was then added again at a trypsin to protein ratio of $1: 100(\mathrm{w} / \mathrm{w})$ for digestion at $37^{\circ} \mathrm{C}$ for $3 \mathrm{~h}$ to complete the digestion cycle.

Western blot. Total proteins $(30 \mu \mathrm{g})$ were isolated from FK228 treated EC109 cells and were separated using SDS-PAGE
(15\% gel), and then transferred to nitrocellulose membranes. After being blocked for $1 \mathrm{~h}$ with the Tris/ $\mathrm{NaCl}$ containing 5\% milk powder, the membranes were probed using an acetylated lysine antibody in a $1: 1,000$ dilution overnight at $4^{\circ} \mathrm{C}$ (cat. no., ab80178; PTM Biolabs, Hangzhou, China). Secondary horseradish peroxidase-labeled anti-goat/rabbit immunoglobulin G (cat. no., 111-035-006; Zhongshan Corp., Beijing, China) was used in a 1:10,000 dilution for $2 \mathrm{~h}$ at room temperature. The protein expression of each sample was normalized by that of GAPDH with Quantity One software (version 4.6.9; Bio-Rad Laboratories, Inc., Hercules, CA, USA).

Affinity enrichment of histone lysine-acetylated peptides. Affinity enrichment was performed for the histone peptides. To enrich the lysine-acetylated peptides, the tryptic peptides were divided into three groups and dissolved in NETN buffer $(100 \mathrm{mM} \mathrm{NaCl}, 1 \mathrm{mM}$ EDTA, $50 \mathrm{mM}$ Tris- $\mathrm{HCl}$ and $0.5 \%$ NP-40, pH 8.0) separately. The peptides were then incubated with pre-washed antibody beads (PTM Biolabs, Hangzhou, China) at $4^{\circ} \mathrm{C}$ overnight with gentle shaking. Next, the beads were washed four times with NETN buffer and twice with double-distilled water. The bound peptides were eluted from the beads using $0.1 \%$ TFA and the eluted fractions were combined and vacuum-dried. The resulting peptides were cleaned using $\mathrm{C}_{18}$ ZipTips (EMD Millipore, Billerica, MA, USA), according to the manufacturer's protocol, prior to liquid chromatography tandem-mass spectrometry (LC-MS/MS) analysis.

LC-MS/MSanalysis. Thepeptides were dissolved in $0.1 \%$ formic acid and loaded directly onto a reversed-phase pre-column (Acclaim PepMap 100; Thermo Fisher Scientific, Inc.). Peptide separation was performed using a reversed-phase analytical column (Acclaim PepMap RSLC; Thermo Fisher Scientific, Inc.) with a linear gradient of 5-35\% solvent B (0.1\% TFA in 98\% acetonitrile) for $30 \mathrm{~min}$ and $35-80 \%$ solvent B for $10 \mathrm{~min}$ at a constant flow rate of $300 \mathrm{nl} / \mathrm{min}$ on an EASY-nLC 1000 UPLC system (Thermo Fisher Scientific, Inc.). The resulting peptides were analyzed using a Q Exactive ${ }^{\mathrm{TM}}$ Plus mass spectrometer (Thermo Fisher Scientific, Inc.). Subsequently, the peptides were subjected to a nanospray ionization source, followed by tandem mass spectrometry (MS/MS) in Q Exactive (Thermo Fisher Scientific, Inc.) coupled online to the UPLC. Intact peptides were detected in the Orbitrap at a resolution of 70,000. The peptides were selected for MS/MS using $27 \%$ normalized collision energy (NCE) with $12 \%$ stepped NCE; ion fragments were detected in the Orbitrap at a resolution of 17,500. A data-dependent procedure that alternated between one MS scan followed by $20 \mathrm{MS} / \mathrm{MS}$ scans was applied for the top 20 precursor ions above a threshold ion count of $3 \times 10^{4}$ in the MS survey scan with $15.0 \mathrm{sec}$ dynamic exclusion. The electrospray voltage applied was $1.8 \mathrm{kV}$. Automatic gain control was used to prevent overfilling of the ion trap; $1 \times 10^{5}$ ions were accumulated for generation of the MS/MS spectra. For the MS scans, the $\mathrm{m} / \mathrm{z}$ scan range was between 350 and 1,600 Da.

Database search. The resulting MS/MS data were processed using MaxQuant with integrated Andromeda search engine (version 1.4.1.2; www.maxquant.org). Tandem mass spectra were searched against the UniProt/Swiss-Protdatabase concatenated with a reverse decoy database. Trypsin/P was 
specified as the cleavage enzyme, allowing up to two missing cleavages, four modifications per peptide and five charges. The mass error was set to $10 \mathrm{ppm}$ for precursor ions and $0.02 \mathrm{Da}$ for fragment ions. Carbamidomethylation on cysteine residues was specified as a fixed modification, and oxidation on methionine residues and acetylation on the $\mathrm{N}$-termini of the proteins were specified as variable modifications. False discovery rate thresholds for the protein, peptide and modification site were specified at $1 \%$. The minimum peptide length was set at 6 . All the other parameters in MaxQuant were set to default values.

Functional enrichment analysis. To further explore the effect of the differentially expressed proteins on the physiological processes in the cells and to identify the internal associations between the differentially expressed proteins, the functions of the differentially expressed proteins were classified and the significance of functional enrichment of Gene Ontology (GO) terms (biological process, cellular component, molecular function), domains and the Kyoto Encyclopedia of Genes and Genomes (KEGG) pathway were analyzed.

Protein annotation, classification and subcellular location prediction. The GO annotation proteome was derived from the UniProt-GOA database (www.ebi.ac.uk/GOA). First, the identifier (ID) of the identified protein was converted into a UniProt ID and then mapped to GO IDs by the protein ID. If identified proteins were not annotated by the UniProt-GOA database, InterProScansoft would be used to annotate the protein's GO function using the protein sequence alignment method. The proteins were then classified by their GO annotation based on three categories: Biological process, cellular component and molecular function. The KEGG database was used to annotate the protein pathways. First, the KEGG online service tool, KAAS, was used to annotate the protein's KEGG database description. The annotation result was then mapped on the KEGG pathway database using the KEGG online service tool, KEGG mapper. Next, WoLFPSORT (https://wolfpsort. hgc.jp/), a subcellular localization predication software, was used to predict the subcellular localization of the proteins. A two-tailed Fisher's exact test was used to statistically analyze the difference among members of protein clusters in specific annotation terms. $\mathrm{P}<0.05$ was considered to indicate a statistically significant difference.

\section{Results and Discussion}

FK228 increased global histone lysine acetylation in the EC109 cells. The viability of the EC109 cells decreased upon treatment with increasing doses of FK228. The cells were maintained in SILAC medium for $24 \mathrm{~h}$ for the lysine acetylation experiments. The global lysine acetylome was detected using western blotting (data not shown).

Next, site-specific identification of histone lysine acetylation in the EC109 cells was carried out. SILAC-based quantitative proteomics was carried out to profile histone lysine acetylation with or without FK228 treatment. A total of 87 lysine acetylation (Kac) sites from the histones and HATs were identified in the present study, of which 25 sites were quantifiable and 19 were quantified with a ratio of $>1.3$ (Fig. 1A). The representative spectra of the histone lysine-acetylated peptides are shown
Table I. Summary of the identified lysine acetylation sites and the quantifiable changes in the EC109 cells treated with FK228.

\begin{tabular}{llc}
\hline Modified site & \multicolumn{1}{c}{$\begin{array}{c}\text { Modified sequence } \\
\text { of peptide }\end{array}$} & $\begin{array}{c}\text { Quantifiable } \\
\text { fold change }\end{array}$ \\
\hline H1K17ac & _APAEK(ac)TP_ & 2.92 \\
H2AK96ac & _EELNK(ac)LLG__ & 1.36 \\
H2AK285ac & _WGADK(ac)CEE_ & 1.39 \\
H2BK12ac & _PAPK(ac)K(ac)GS_ & 130.67 \\
H2BK13ac & _PAPK(ac)K(ac)GS_ & 130.67 \\
H2BK16ac & _GSK(ac)K(ac)AVTK_ & 130.67 \\
H2BK17ac & _GSK(ac)K(ac)AVTK_ & 130.67 \\
H2BK18ac & _K(ac)GSK(ac)K(ac)AV_ & 130.67 \\
H2BK21ac & _K(ac)K(ac)AVTK(ac)_ & 130.67 \\
H2BK22ac & _K(ac)K(ac)AVTK(ac) _ & 130.67 \\
H2BK44ac & _YSVYVYK(ac)VLK_ & 1.51 \\
H2BK109ac & _GELAK(ac)HAVS_ & 1.39 \\
H3K24ac & _QLATK(ac)AAR__ & 5.94 \\
H3K57ac & _QK(ac)STELLIR__ & 1.49 \\
H3K80ac & _AQDFK(ac)TDR_- & 1.14 \\
H3K123ac & _PK(ac)DIQLAR_- & 1.4 \\
H4K32ac & _IQGITK(ac)PAIR_ & 1.23 \\
H4K60ac & _GVLK(ac)VFLEN_- & 1.19 \\
H4K80ac & _K(ac)TVTAMD__ & 1.59 \\
H4K92ac & _MDVVYALK(ac)R_ & 1.18 \\
\hline
\end{tabular}

$\mathrm{H}$, histone; $\mathrm{K}$, lysine; ac, acetylation.

in Fig. 1B-F, including the spectra for the H3K24ac, H2K14ac, $\mathrm{H} 2 \mathrm{~K} 17 \mathrm{ac}$ and H2K22ac peptides. Notably, no downregulated Kac sites were quantified in the present study. Furthermore, the 62 unquantified Kac sites were only identified in the FK228-treated cells. The distinct profiles of histone lysine acetylation following FK228 treatment revealed the effects of FK228 on the EC109 cells. The sequences of the identified lysine-acetylated peptides in the core histones and the corresponding quantitative changes in the Kac profiles in response to FK228 treatment are summarized in Table I.

The abundance of the identified Kac sites in the EC109 cells was compared. The relative abundance was calculated based on the ratio of the peak areas of the same parent ions labeled by the light and heavy isotopes in the MS spectra. Among these Kac sites, H2BK12ac, H2BK13ac, H2BK16ac, H2BK17ac, H2BK18ac H2BK21ac and H2BK22ac exhibited significant differential abundance sites, with a 130 -fold difference between the FK228-treated and -untreated cells. To the best of our knowledge, the present study is the first to identify these Kac sites in EC109 cells and their functions require further investigation in future studies. In the acetylation site of H3K24, its abundance in the EC109 cells exposed to FK228 was increased 5.9-fold compared with that observed in the EC109 cells that were not treated with FK228. The acetylation level in H1K17ac was also increased 2.92-fold in the EC109 cells following FK228 treatment. These results provide a comprehensive identification of sites of histone 

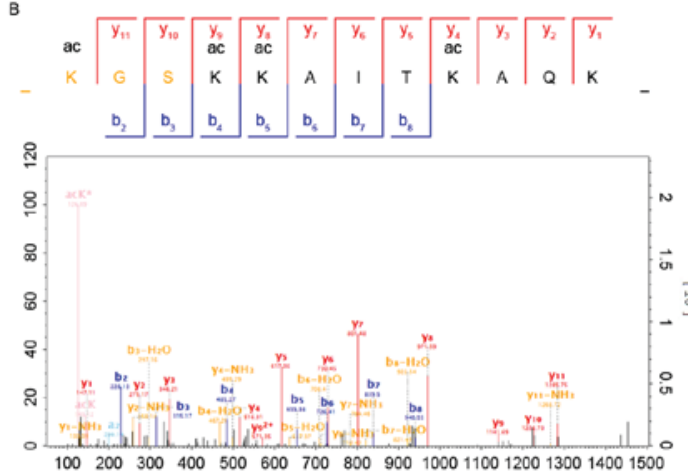

D
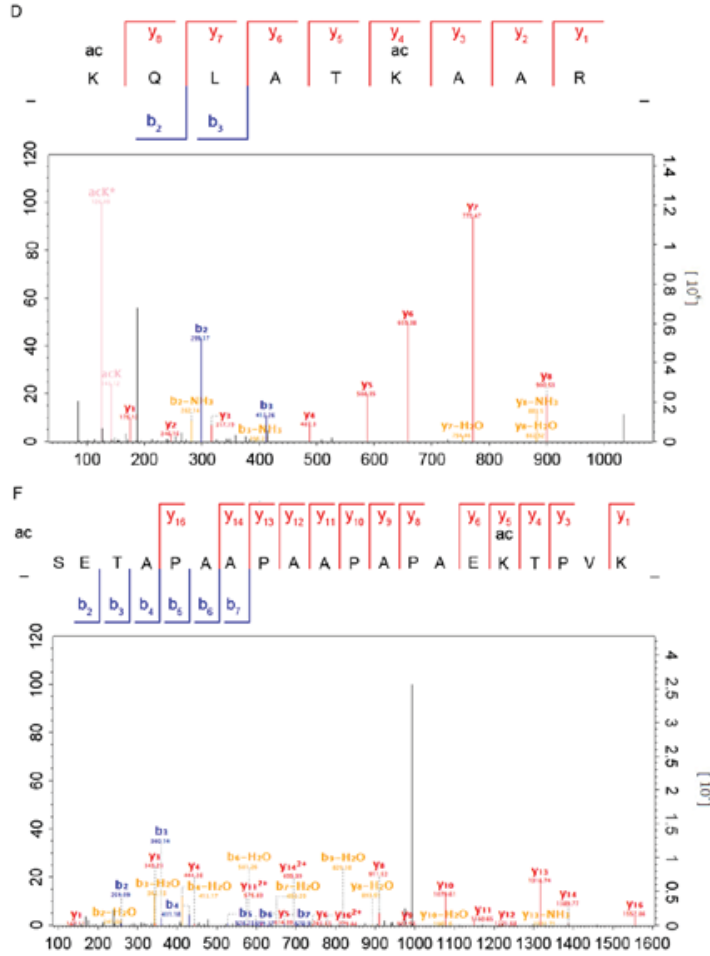
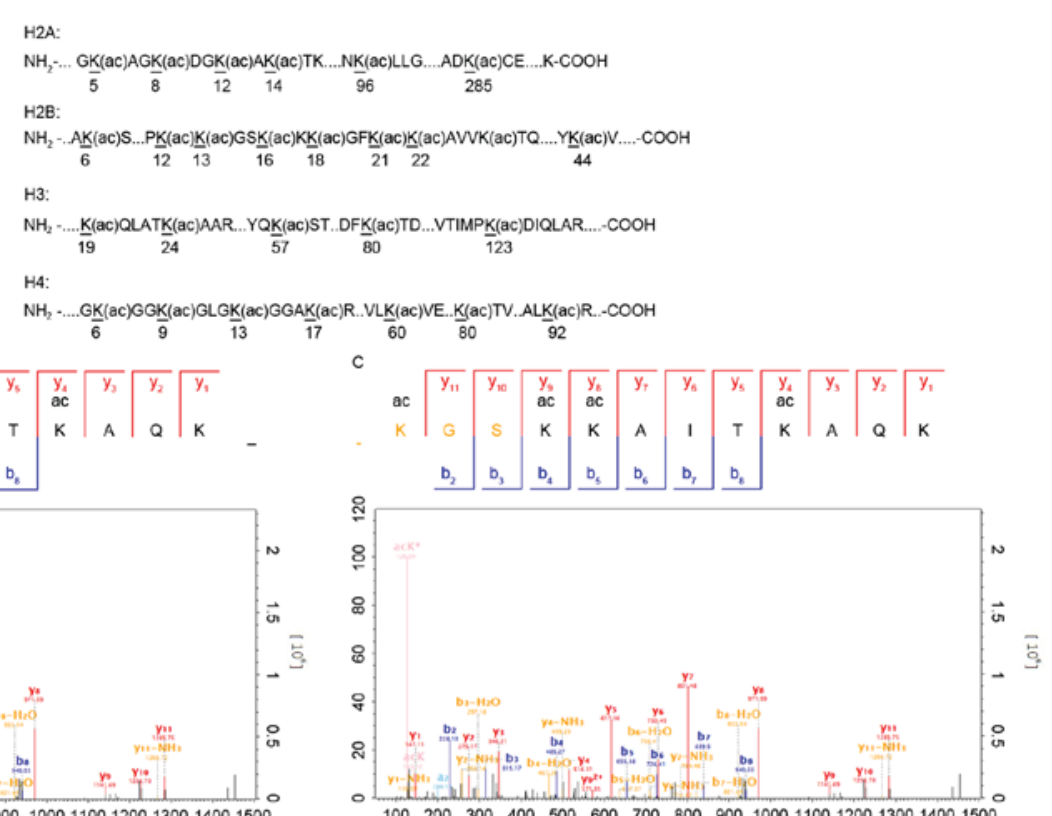

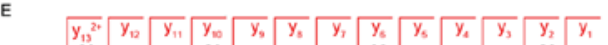
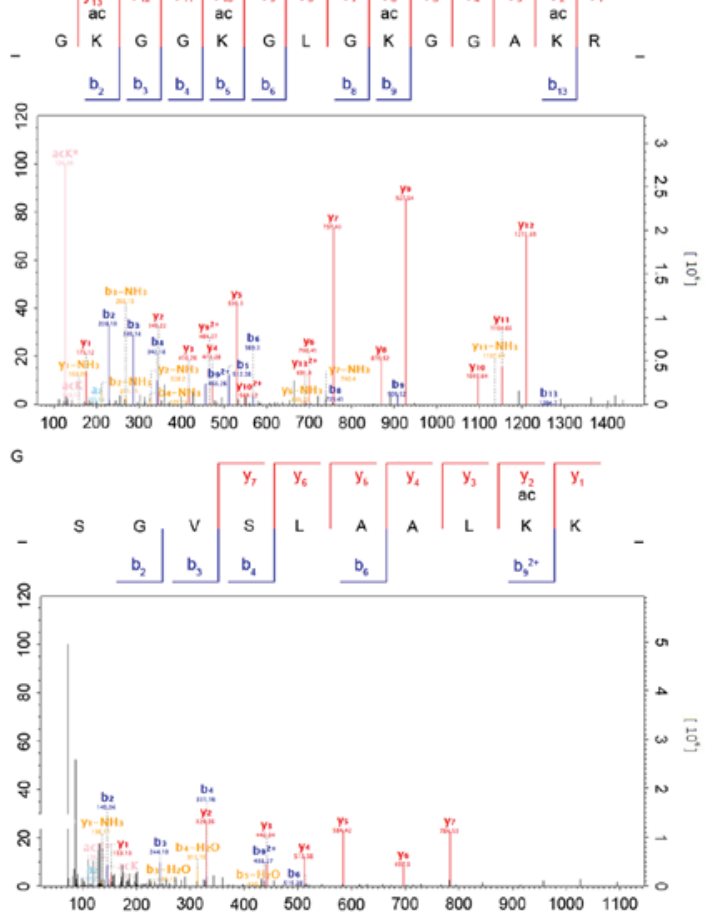

Figure 1. Identification and quantification of Kac sites in EC109 cells. (A) Illustration of the identified Kac sites in EC109 cells treated with FK228. The identified sites are numbered and highlighted. Tandem mass spectrometry spectra of tryptic peptide on: (B) H2BK13-ac peptide_SAPAPK(ac)K(ac)GS; (C) H2BK17-ac peptide_GSK(ac)K(ac)AVTK; (D) H3K24-ac peptide_QLATK(ac)AAR; (E) H4K13-ac peptide_GK(ac)GGK(ac)GLGK(ac)GGAK(ac)R; (F) H1K17-ac peptide_PAPAEK(ac)TPVK; (G) H1K63-acpeptide_SGVSLAALK(ac)K.H, histone; K, lysine; ac, acetylated.

lysine acetylation and reveal the potential epigenetic mechanism underlying the effects of FK228 in the EC109 cells. The results of the present study suggest that FK228-based therapy may have potential for use in treating ESCC.

FK228, one of the powerful HDAC inhibitors, inhibits class I HDAC enzymes more than class II HDAC enzymes. It inhibits the removal of acetyl groups from the lysine residues of N-terminal histone tails (15) and also alters the acetylation of other nuclear and cytoplasmic proteins including heat-shock protein 90 chaperone proteins (11). Histone acetylation states are crucial to a number of cellular processes; for example, the cycles of acetylation and deacetylation may prepare the genes including oncogenes and tumor suppressor genes for future activation. The present study observed the distinct effects of FK228 on histone lysine acetylation in the EC109 cells. Almost all the quantifiable lysine-acetylated sites were in the N-termini of core histones, suggesting the profound epigenetic modulation mechanism underlying the effect FK228 in the EC109 cells. Among the quantifiable lysine-acetylated sites, 22 sites were novel acetylated sites and seven sites significantly increased their acetylation level in response to FK228 exposure. Further studies should focus on the lysine-acetylated sites that demonstrated significant alterations 
Fisher's exact Test P-value

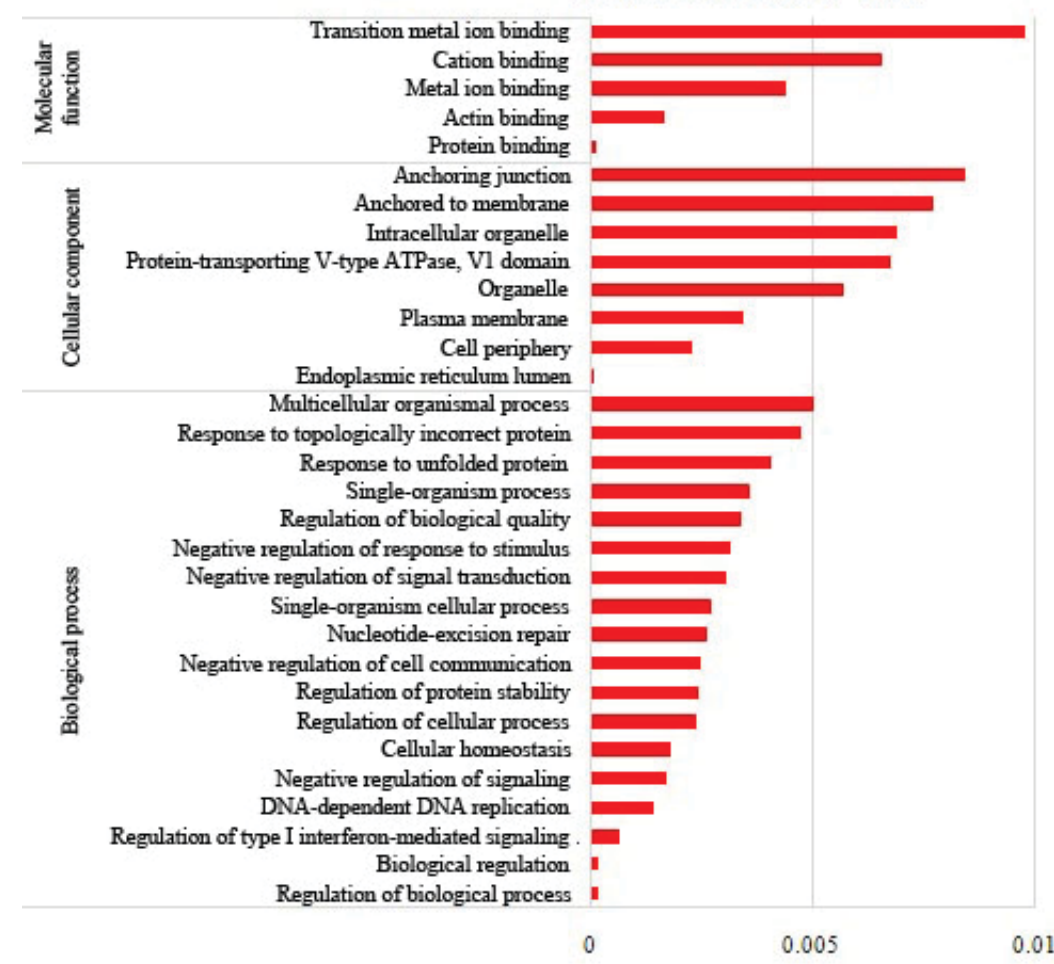

Figure 2. Gene Ontology-based enrichment in the upregulated proteins, as determined by functional enrichment analysis.

in histones to explore the epigenetic mechanism underlying the effect of FK228 in the EC109 cells.

Proteome profile of the EC109 cells treated with FK228. HATs, which acetylate the lysine residues of histone proteins, facilitate the access of numerous transcriptional factors to the DNA and consequently activate the expression of the target genes. Therefore, the increased or decreased level of histone lysine acetylation may correspondingly alter the proteome of the EC109 cells. To confirm this, quantitative proteomics was performed to profile the proteome of the EC109 cells with or without FK228 exposure. A total of 5,279 proteins were identified in the EC109 cells, of which 3,515 proteins were quantified. Of these 3,515 quantifiable proteins, 675 proteins demonstrated increased histone lysine acetylation levels and 186 proteins demonstrated decreased levels, which suggested that the distinct profiles of histone lysine acetylation induced by FK228 treatment may lead to distinct proteomic profiles.

Biological functions of the quantified proteins in the EC109 cells treated with FK228. To gain a better understanding of the function and characteristics of the quantified proteins, the functions or features of the proteins from several distinct categories, including GO terms, domains, pathways and subcellular localization were noted. The proteins were classified by GO annotation on the basis of three categories: Biological process, cellular component and molecular function. In the biological process category (Fig. 2), the proteins associated with the regulation of biological processes, biological regulation, regulation of the type I interferon-mediated signaling pathway, regulation of protein stability, regulation of cellular processes and negative regulation of signal transduction were upregulated, whereas those associated with cellular hormone metabolism, hormone metabolism, carboxylic acid metabolism, lipid metabolism, oxoacid metabolism, steroid metabolism, hormone biosynthesis and organic acid metabolism were downregulated (Fig. 3). These results implied that FK228 treatment affected biological regulation and hormone metabolism in the EC109 cells.

Enrichment analysis of the molecular functions revealed that proteins associated with protein binding, actin binding, metal ion binding and cation binding were upregulated (Fig. 2), whereas those associated with oxidoreductase activity and aldo-ketoreductase (nicotinamide-adenine dinucleotide phosphate) activity were downregulated (Fig. 3). These results suggested that the proteins involved in binding functions, which may regulate enzyme activity, were enriched following FK228 exposure. Analysis by cellular component revealed that the endoplasmic reticulum lumen was the major cellular component in the EC109 cells that responded to the acetylation changes. Therefore, the GO analysis suggested that the enriched biological functions were associated with lysine acetylation, implying an HDAC-inhibiting role of FK228 in the EC109 cells.

Domain structure is a major functional element in proteins. To investigate the domain features of the enriched proteins, the proteins that were enriched upon FK228 treatment were analyzed using Interpro domain enrichment analysis. The results revealed that protein domains including the PDZ domain, zinc finger domain and EF hand domain were enriched following FK228 treatment (Fig. 4). 
Fisher's exact Test P-value

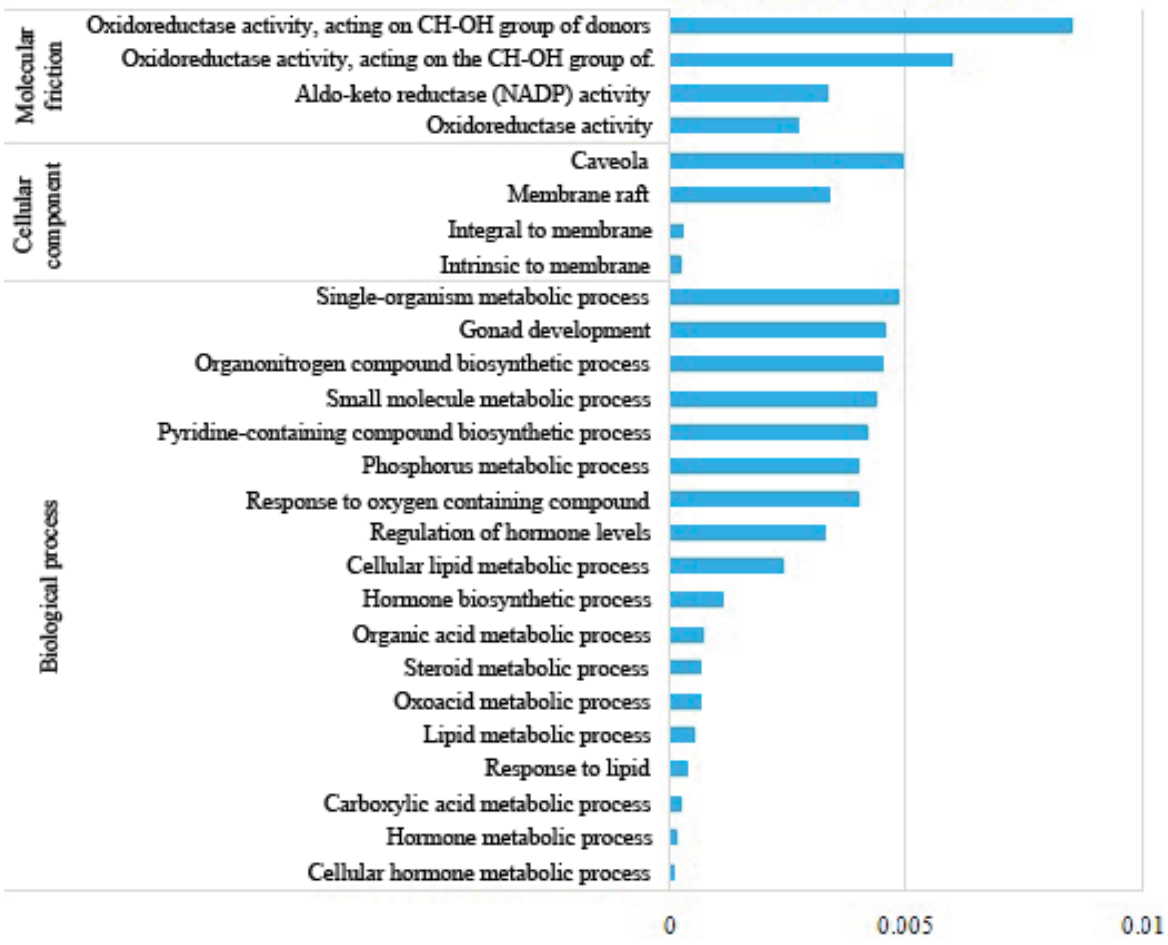

Figure 3. Gene Ontology-based enrichment in the downregulated proteins, as determined by functional enrichment analysis. Enrichment analysis of protein domains, cellular pathways and protein complexes in the EC109 cells following FK228 treatment.

Fisher's exact Test P-value

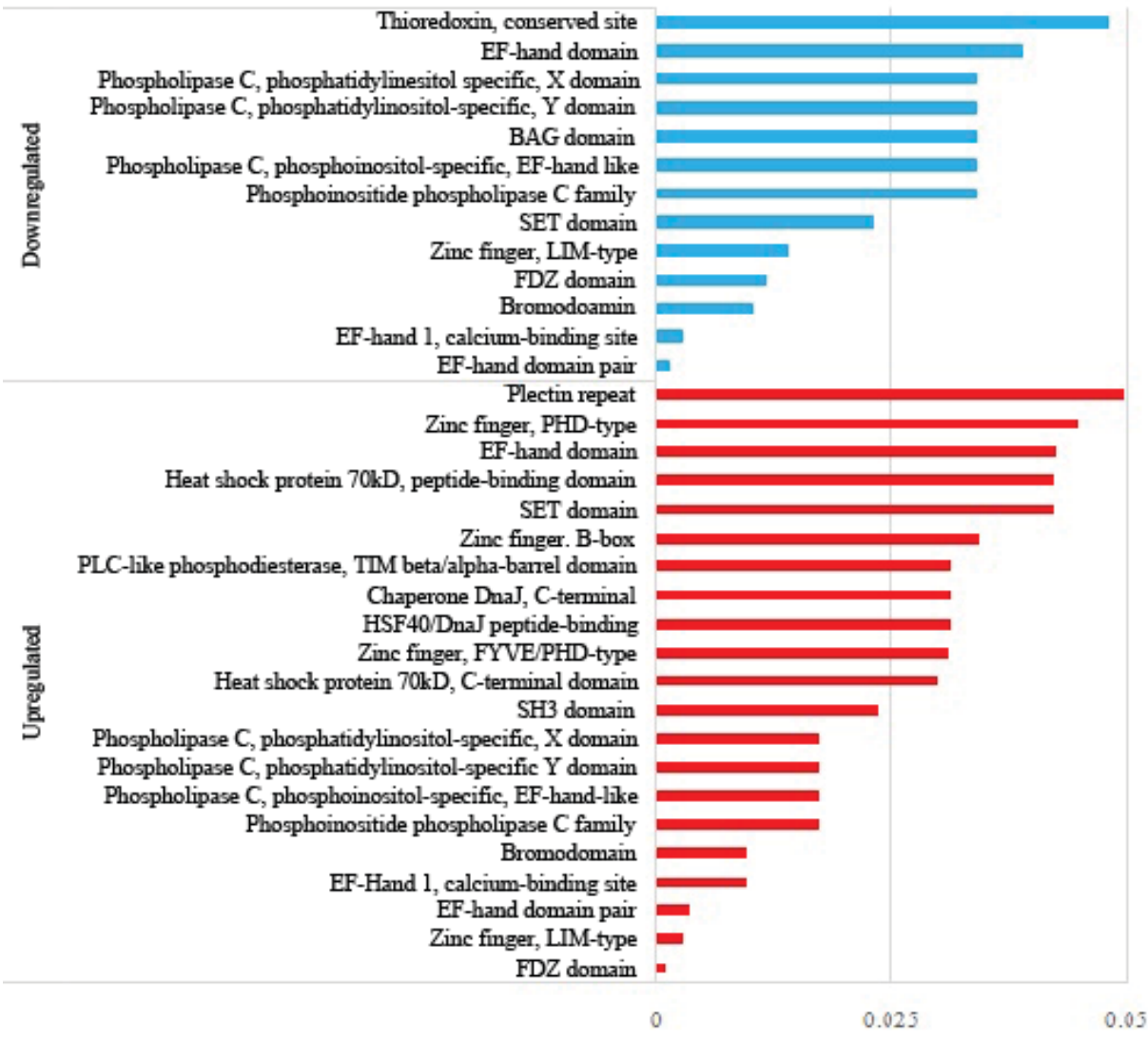

Figure 4. Domains enriched in the up- and downregulated proteins, as determined by functional enrichment analysis. The pathways in green were downregulated and the pathways in red were upregulated. 


\section{Fisher's exact Test P-value}

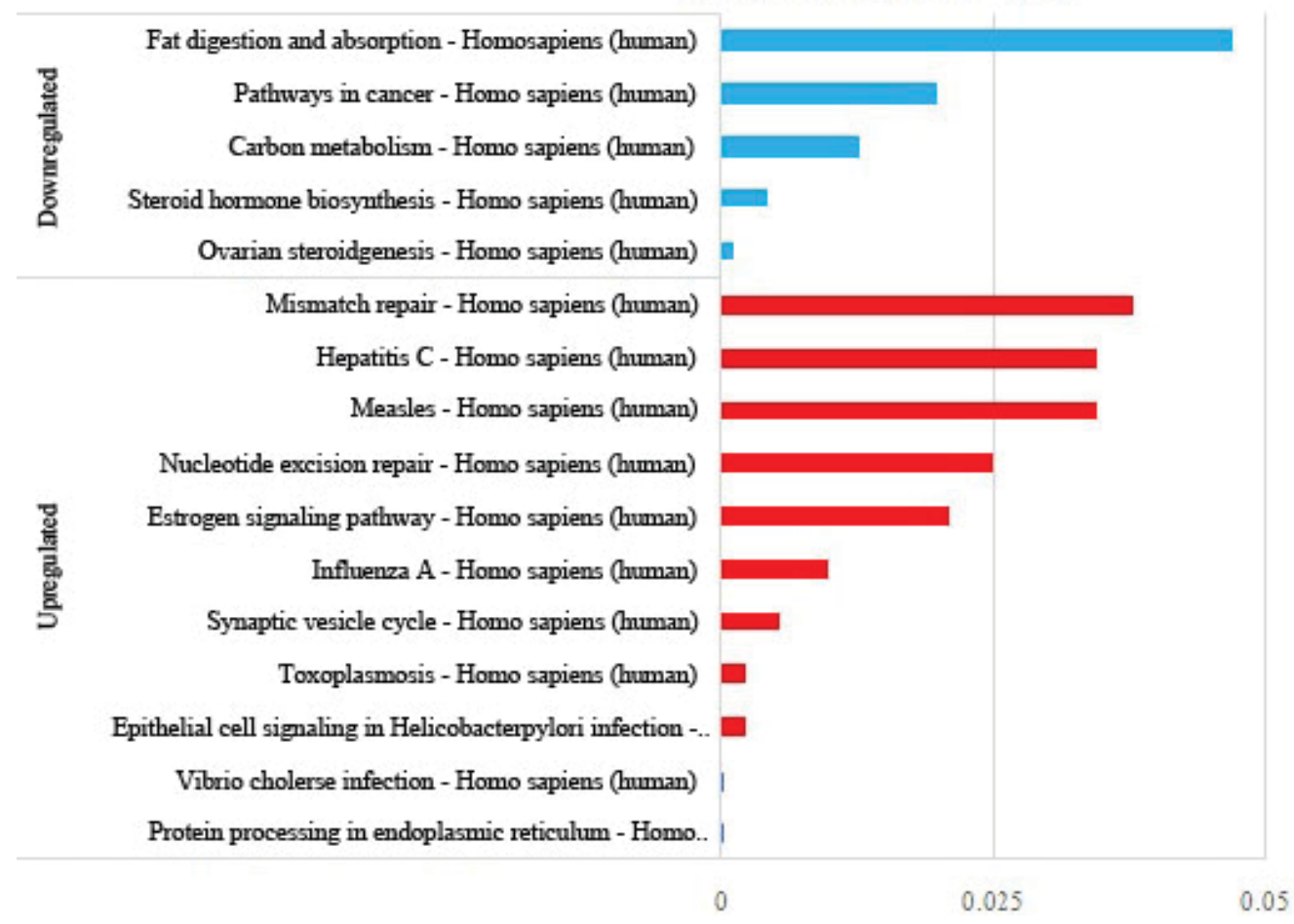

Figure 5. Pathways obtained by Kyoto Encyclopedia of Genes and Genomes pathway enrichment analysis. The pathways in green were downregulated and the pathways in red were upregulated.

Next, pathway clustering analysis of the FK228-responsive proteome from the KEGG database was performed to examine the cellular pathways affected by FK228 treatment (Fig. 5). The results revealed that protein processing in the endoplasmic reticulum, Vibrio cholerae infection, epithelial cell signaling in Helicobacter pylori infection and toxoplasmosis were the most distinct signaling pathways exhibiting increased protein levels in the FK228-treated cells. This suggested a role for FK228 in these signaling pathways. However, the protein level in the signaling pathway of ovarian steroidogenesis and steroid hormone biosynthesis was decreased in the FK228-treated cells. These results were in agreement with those observed in biological process regulation, where the hormone metabolism signaling pathway and others were downregulated.

In the present study, quantitative proteomics and bioinformatic analysis were used to characterize the global profile of histone lysine acetylation and the quantitative proteomic changes in EC109 cells treated with FK228. A number of Kac sites including novel acetylation sites were identified in the EC109 cells. As histone lysine acetylation regulates the activation of gene transcription by regulating the binding of histone proteins to DNA, the different levels of histone lysine acetylation observed in the present study may affect the expression profile of the proteome. Bioinformatic analysis further revealed that FK228 regulates protein expression by affecting multiple biological signaling pathways and protein complexes in the EC109 cells. The results of the present study improve current knowledge of the therapeutic properties and the molecular mechanism underlying the effects of FK228 as an HDAC inhibitor in EC109 cells.

\section{Acknowledgements}

Not applicable.

\section{Funding}

The present study was supported by grants from the Zhejiang Cancer Hospital (grant no. 2013Y012) and the Medical and the Health Science Project of Zhejiang Province (grant no. 2015KYA033).

\section{Availability of data and materials}

The datasets used and analyzed during the present study are available from the corresponding author on reasonable request.

\section{Authors' contributions}

ZP analyzed and interpreted the data, and was a major contributor in writing the manuscript. MW performed experiments and contributed to data analysis. ZY performed experiments and contributed materials and analysis tools. SZ contributed significantly to data analysis and manuscript preparation. XX was responsible for conception of the study, and manuscript revision. All authors read and approved the final manuscript.

\section{Ethics approval and consent to participate}

Not applicable. 


\section{Consent for publication}

Not applicable.

\section{Competing interests}

The authors declare that they have no conflict of interest.

\section{References}

1. Layke JC and Lopez PP: Esophageal cancer: A review and update. Am Fam Physician 73: 2187-2194, 2006.

2. Qi YJ, Chao WX and Chiu JF: An overview of esophageal squamous cell carcinoma proteomics. J proteomics 75: 3129-3137, 2012.

3. Emanuele S, Lauricella M and Tesoriere G: Histone deacetylase inhibitors: Apoptotic effects and clinical implications (Review). Int J Oncol 33: 637-646, 2008

4. Glaser KB: HDAC inhibitors: Clinical update and mechanism-based potential. Biochem Pharmacol 74: 659-671, 2007.

5. Fukuda H, Sano N, Muto S and Horikoshi M: Simple histone acetylation plays a complex role in the regulation of gene expression. Brief Funct Genomic Proteomic 5: 190-208, 2006.

6. Marks PA, Richon VM, Breslow R and Rifkind RA: Histone deacetylase inhibitors as new cancer drugs. Curr Opin Oncol 13 : 477-483, 2001.

7. Jain S, Jirau-Serrano X, Zullo KM, Scotto L, Palermo CF Sastra SA, Olive KP, Cremers S, Thomas T, Wei Y, et al: Preclinical pharmacologic evaluation of pralatrexate and romidepsin confirms potent synergy of the combination in a murine model of human T-cell lymphoma. Clin Cancer Res 21: 2096-2106, 2015.
8. Stühmer T, Arts J, Chatterjee M, Borawski J, Wolff A, King P, Einsele H, Leo E and Bargou RC: Preclinical anti-myeloma activity of the novel HDAC-inhibitor JNJ-26481585. Br J Haematol 149: 529-536, 2010.

9. VanderMolen KM, McCulloch W, Pearce CJ and Oberlies NH Romidepsin (Istodax, NSC 630176, FR901228, FK228, depsipeptide): A natural product recently approved for cutaneous T-cell lymphoma. J Antibiot (Tokyo) 64: 525-531, 2011.

10. Pellegrini C, Dodero A, Chiappella A, Monaco F, Degl'Innocenti D, Salvi F, Vitolo U, Argnani L, Corradini P and Zinzani PL; Italian Lymphoma Foundation (Fondazione Italiana Linfomi Onlus, FIL): A phase II study on the role of gemcitabine plus romidepsin (GEMRO regimen) in the treatment of relapsed/refractory peripheral T-cell lymphoma patients. J Hematol Oncol 9: 38, 2016.

11. Yu X, Guo ZS, Marcu MG, Neckers L, Nguyen DM, Chen GA and Schrump DS: Modulation of p53, ErbB1, ErbB2, and Raf-1 expression in lung cancer cells by depsipeptide FR901228. J Natl Cancer Inst 94: 504-513, 2002.

12. Archer SY, Meng S, Shei A and Hodin RA: p21(WAF1) is required for butyrate-mediated growth inhibition of human colon cancer cells. Proc Natl Acad Sci USA 95: 6791-6796, 1998.

13. Kwon HJ, Kim MS, Kim MJ, Nakajima H and Kim KW: Histone deacetylase inhibitor FK228 inhibits tumor angiogenesis. Int J Cancer 97: 290-296, 2002.

14. Insinga A, Monestiroli S, Ronzoni S, Gelmetti V, Marchesi F, Viale A, Altucci L, Nervi C, Minucci S and Pelicci PG: Inhibitors of histone deacetylases induce tumor-selective apoptosis through activation of the death receptor pathway. Nat Med 11: 71-76, 2005.

15. Kuo MH and Allis CD: Roles of histone acetyltransferases and deacetylases in gene regulation. Bioessays 20: 615-626, 1998. 